\title{
Savoirs techniques locaux et pratiques : la conduite des troupeaux aux pâturages (Ouest du Burkina Faso)
}

\author{
Éric Vall ${ }^{1}$, Mohamadoun Amadou Diallo \\ 1 Zootechnicien, CIRAD/CIRDES, Bobo-Dioulasso 01, Burkina Faso \\ 2 Zootechnicien, LERNSE/UPB, CIRDES, Bobo-Dioulasso 01, Burkina Faso
}

\begin{abstract}
La revue NSS a souvent examiné la place des savoirs traditionnels dans les rapports entre sociétés et sciences. La revalorisation de ces savoirs entre dans les nouvelles façons de penser des recherches sur le terrain, notamment dans les sociétés du Sud. Cet article s'attache ainsi à la construction de connaissances scientifiques à partir de savoirs techniques locaux. En distinguant savoirs locaux et pratiques, les auteurs introduisent un cadre d'analyse rigoureux qu'ils illustrent à travers l'étude des conduites de troupeaux aux pâturages dans un territoire villageois africain. Leur recherche innove surtout par l'adoption des concepts et des mots locaux relatifs aux cadres environnementaux des savoirs et des pratiques. Cette mobilisation des langues locales comme outil de connaissance scientifique ouvre un débat entre agronomes mais aussi entre agronomes et sciences sociales.
\end{abstract}

La Rédaction

\section{Mots-clés :}

pratiques ;

savoirs locaux;

systèmes

agropastoraux; recherche-action; Burkina Faso

\section{Keywords:}

practices;

local knowledge; agro-pastoral systems; action research; Burkina Faso

\begin{abstract}
Résumé - Partant d'une expérience de recherche en partenariat, conduite sur le territoire de Koumbia-Waly (Burkina Faso), visant à établir des règles de gestion concertée des espaces agro-sylvo-pastoraux, l'article montre comment les auteurs ont cherché à rendre intelligibles les pratiques des éleveurs par une analyse basée sur la prise en compte des savoirs locaux. Les références sur les savoirs locaux et les pratiques ont été élaborées à partir d'enquêtes originales, complétées par des observations directes des troupeaux au pâturage. Les savoirs techniques relatifs au calendrier et aux unités pastorales ont été caractérisés sur le plan structurel et fonctionnel. L'analyse de la conduite au pâturage en référence aux savoirs locaux a permis de comprendre les logiques individuelles et de dégager des règles communes aux acteurs. Dans la discussion, les auteurs reviennent sur l'apport des savoirs locaux pour l'analyse des pratiques et pour l'intervention sur celles-ci.
\end{abstract}

\begin{abstract}
Analysis of practices using local technical knowledge: the case of livestock grazing management in western Burkina Faso. Starting from a participative research whose purpose was to design rules for the collective management of agro-pastoral areas in the villages of Koumbia and Wally (Burkina Faso), the paper shows that breeder practices may be understood through analyses that take into account local knowledge. The study was based on a sample of ten stockbreeders. The paper sets out the framework for the characterization of the breeders' local knowledge and practices; the reference data on local knowledge and practices were derived from original investigations and direct observations of grazing livestock. The stockbreeders' knowledge on pastoral schedules (5 periods) and on pastoral land (8 main units) was structurally and functionally characterized. A description of actual grazing management practices of two stockbreeders was elaborated using the analysis framework. This helped to understand individual logics by highlighting circumstantial practices and rules shared by the stockbreeders. One result of the study was to produce a list of main and secondary grazing areas identified by the breeders, to be preserved and managed as a priority. In the discussion the authors highlight the interest of local knowledge in analysing practices and contributing to their improvement.
\end{abstract}

Auteur correspondant : É. Vall, eric.vall@cirad.fr 


\section{Introduction}

\section{Contexte de l'étude}

Dans les zones cotonnières de l'Afrique de l'Ouest, l'agriculture et l'élevage étaient autrefois pratiqués par des populations généralement distinctes. Le faible niveau de pression anthropique permettait une coexistence relativement pacifique des deux activités, car les besoins d'espaces des producteurs pour les cultures et les pâturages étaient satisfaits. L'utilisation séquentielle des zones agricoles (culture en saison des pluies, vaine pâture en saison sèche) ne posait pas de problème, car les agriculteurs possédaient peu de bétail.

Ces vingt dernières années, l'augmentation de la population rurale a entraîné une forte extension des champs cultivés au détriment des parcours naturels, la mise en culture continue des espaces agricoles (abandon de la jachère), l'intensification de l'agriculture ${ }^{1}$ (traction animale, engrais, pesticides) et le développement de l'élevage par les agriculteurs (animaux de trait, puis cheptel de rente). Les éleveurs ont maintenu des systèmes d'élevage fondés sur une exploitation séquentielle des espaces agro-sylvopastoraux sans réelle intensification. Ils ont poursuivi l'élevage de grands troupeaux de bovins et ont développé une agriculture de subsistance (maïs, sorgho), parfois le coton. Ces logiques d'extension des cultures et du cheptel ont entraîné une compétition sur les espaces agrosylvo-pastoraux villageois et, en l'absence de systèmes de régulation adaptés à un niveau de pression anthropique élevé, la conduite des troupeaux au pâturage pose de plus en plus de problèmes et les conflits agropastoraux se sont multipliés (Vall et al., 2006).

Les producteurs des villages de Koumbia et de Waly (zone cotonnière de l'Ouest du Burkina Faso) sont confrontés à cette problématique (Encadré 1). Ils sont conscients des problèmes, mais ne détiennent pas la solution appropriée pour les résoudre et instaurer une réelle complémentarité entre l'agriculture et l'élevage afin de sécuriser ce dernier, d'accroître les productions agropastorales au bénéfice de tous et de préserver les ressources agro-sylvopastorales pour les générations à venir.

\section{Une initiative commune pour répondre à la problématique locale}

En 2005, les producteurs de Koumbia et de Waly et une équipe de chercheurs (Cirad, Cirdes) ont décidé de travailler ensemble pour préciser la problématique agropastorale locale, identifier les solutions, les mettre en œuvre et les évaluer. Cette initiative a été conduite à travers un dispositif de recherche en partenariat, portée par le soutien scientifique et financier de deux projets

\footnotetext{
${ }^{1}$ Encouragée par les sociétés cotonnières pour le développement du coton.
}

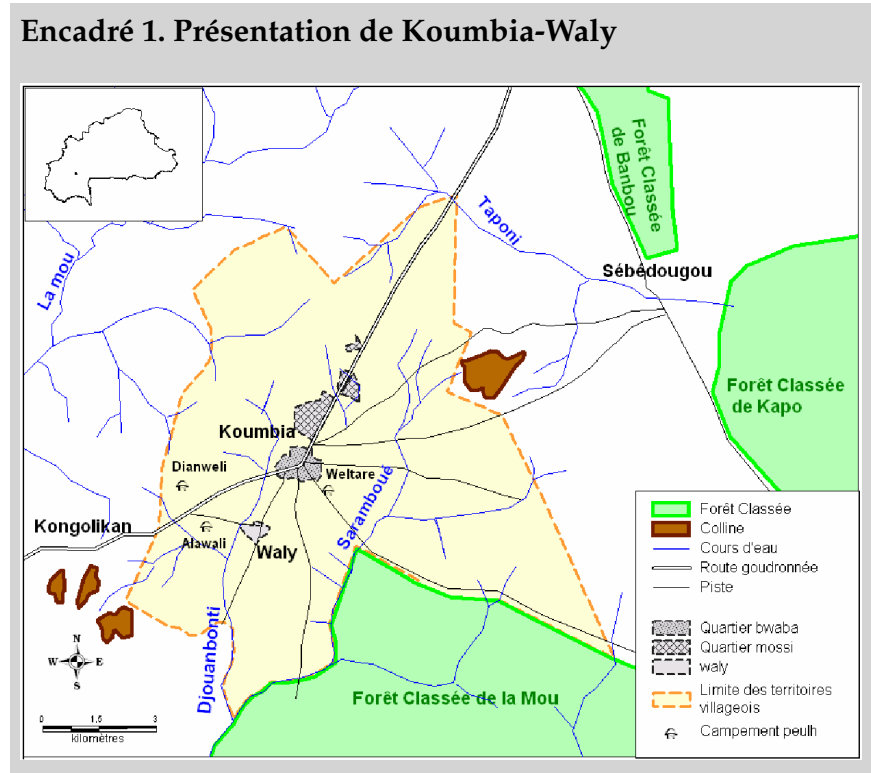

Le territoire de Koumbia-Waly, situé en zone cotonnière de l'Ouest du Burkina, s'étend sur 9100 ha et compte environ 6000 habitants (autochtones : Bwaba; migrants : Mossi, Peuls). Ce village se caractérise par un niveau de pression anthropique élevé sur les ressources agro-sylvo-pastorales $\left(66 \mathrm{hab} / \mathrm{km}^{2}\right.$, $48 \mathrm{UBT}^{*} / \mathrm{km}^{2}$, emprise agricole de $53 \%$ ). Il est composé de deux zones d'habitations principales (Koumbia et Waly) et de trois campements d'éleveurs peuls à la périphérie de celles-ci (Alawali, Weltare, Dianweli).

Les systèmes de production sont familiaux, basés sur la production de coton (53\% de l'assolement), de maïs (36 \% de l'assolement) et de bovins. Les unités de production (UP) se répartissent entre :

- de très petites unités (3 ha cultivés), non équipées en traction animale (13\% des UP);

- une majorité d'unités (71\%) où l'agriculture est dominante (6 à 14 ha cultivés), équipées d'une ou deux paires de bœufs ;

- un noyau d'agro-éleveurs peu nombreux (7 \% des UP), issus des agriculteurs, bien équipés (4 à 10 bovins de trait), cultivant de grandes surfaces ( 8 à 35 ha) et ayant développé un noyau d'élevage de 20 à 30 bovins ;

- des unités dominées par l'élevage bovin (25 à 105 têtes) où l'agriculture n'a pas de visée commerciale marquée (maïs, sorgho sur 2 à 3 ha); elles sont conduites par des Peuls.

* UBT : unité de bétail tropical (1 UBT = 1 bovin de $250 \mathrm{~kg}$ ).

(Agri-élevage de DURAS et ATP CIROP du Cirad²). La démarche de recherche en partenariat comprend quatre composantes : 1) gouvernance du partenariat ;2) diagnostic et problématisation des situations ; 3 ) élaboration des hypothèses et des solutions ; 4) mise en œuvre des solutions et évaluation. Cet article sera focalisé sur la partie diagnostic de la composante 2. Les composantes 3 et 4 ,

\footnotetext{
2 ATP CIROP : action thématique programmée «Conception des innovations et rôle du partenariat »; projet DURAS : promotion du développement durable dans les systèmes de recherche agricole du Sud.
} 
mises en œuvre avec le soutien du projet Fertipartenaires (UE) à partir de 2008, ne seront pas exposées.

Une étude préliminaire, conduite selon une approche systémique classique, a permis de cerner la problématique agropastorale locale (Blanchard, 2005). Selon cette étude, la surface totale cultivée (environ 5000 ha) additionnée à la surface de pâturage nécessaire pour couvrir les besoins alimentaires du bétail $\left(9000 \mathrm{ha}^{3}\right)$ dépasse la surface totale du territoire villageois (9 100 ha). Les deux activités ne peuvent être conduites sur des espaces séparés. De plus, la majorité des producteurs étant à la fois cultivateurs et détenteurs de troupeaux, l'exploitation partagée et séquentielle des espaces requiert des règles reconnues par l'ensemble de la communauté villageoise. Or, le déficit de gestion concertée des espaces agro-sylvo-pastoraux conduit à de multiples conflits. Lors de la restitution de cette première étude, les acteurs de terrain et les scientifiques se sont accordés sur les processus en cours et leurs conséquences si rien n'était fait.

Pour agir face à cette situation, un partenariat a été organisé afin d'impliquer l'ensemble des parties prenantes dans l'identification des problèmes et dans l'élaboration des solutions. Ce travail a abouti à la mise en place d'un comité de coordination villageois représentatif, chargé de réfléchir au problème suivant : comment impliquer les populations, leurs représentants et les services techniques pour faciliter et sécuriser la mobilité du bétail, élaborer des modalités de gestion durable des ressources agro-sylvo-pastorales et réduire les conflits entre agriculteurs et éleveurs? Ce comité a convenu de travailler sur l'élaboration d'une convention locale qui précisera les règles d'utilisation des espaces agro-sylvo-pastoraux du territoire de Koumbia-Waly et les modalités de règlement des conflits, en accord avec les pratiques pastorales locales et les textes de loi, en vue d'une exploitation durable des espaces.

L'élaboration d'une telle convention nécessite une bonne connaissance des pratiques pastorales locales et de leurs fondements. C'est la raison pour laquelle une étude approfondie des modes de conduite des troupeaux de bovins au pâturage a été mise en œuvre (Diallo, 2006).

\section{Pourquoi recourir aux savoirs techniques locaux dans l'analyse des pratiques?}

Énoncés écrits ou oraux partagés par un groupe social, les savoirs locaux s'insèrent dans un ensemble de visions des choses et du monde (croyances...), de connaissances établies (sciences, philosophies...), propres au groupe social et à l'époque (Dupré, 1991; Scoones et Thompson, 1999). Ils touchent tous les domaines de la vie. Dans le contexte de l'étude, les savoirs techniques locaux, proches de la notion de savoir-faire, portent sur l'agriculture,

3 À raison de 2 ha/unité de bétail tropical/an. l'élevage, l'environnement, la santé humaine et animale, l'économie, la gestion et la vie domestique et ils « règlent les pratiques »(Olivier de Sardan, 1996). Dans une perspective $d$ 'intervention sur les pratiques, il est donc important que les agronomes essaient de les prendre en compte dans leurs analyses, ce qui ne va pas de soi.

Les récentes études sur les pratiques de conduite des troupeaux au pâturage en zone agropastorale (Petit, 2000 ; Ickowicz et Mbaye, 2001; Botoni, 2003; Kiéma, 2007; Augusseau, 2007) ont produit des références reconnues dans la sphère scientifique, mais elles restent cependant disjointes de la réalité des praticiens, car elles prennent peu en compte la manière dont ceux-ci pensent et exploitent leur territoire pastoral. Les savoirs locaux sont très souvent occultés et l'étude des pratiques de pâturage n'est que rarement référée aux représentations des acteurs locaux relatives au temps et à l'espace (Kagoné, 2000).

Les références sur les pratiques sont généralement élaborées sur des entités définies a priori par les scientifiques (saisons, unités pastorales), qui ne correspondent pas tout à fait aux entités réellement utilisées par les praticiens dans la conduite de leurs activités. Or, dans une perspective d'intervention, les références élaborées doivent être compréhensibles à la fois par les praticiens et par les scientifiques pour être " actionnables » (Avenier et Schmitt, 2007). Elles doivent rendre les pratiques intelligibles par tous les acteurs de la recherche, éclairer les options de transformation possibles et leur faisabilité (Liu, 1997).

Pour rendre compte de la manière dont les praticiens se représentent les choses et fondent leurs décisions, Darré et al. (2004) ont proposé une méthode d'analyse de leur discours. Mais cette approche, développée par des sociologues, nous a semblé difficile à mettre en œuvre par des zootechniciens sur un échantillon significatif de producteurs et, qui plus est, dans un environnement plurilingue (bwamu, moore, fulfulde).

Nous proposons donc d'élaborer et de tester un cadre $\mathrm{d}$ 'analyse visant à donner du sens aux pratiques en nous basant sur une étude préalable des savoirs techniques locaux dans différents domaines, complétée par des observations directes des pratiques et des séances de restitution/validation aux acteurs de terrain. Cette approche des pratiques à partir des savoirs techniques locaux vise à extraire les fondements logiques qui les règlent et les sous-tendent, afin de les rendre intelligibles.

À partir des travaux menés sur les pratiques de conduite des troupeaux au pâturage des éleveurs et agro-éleveurs de Koumbia-Waly (Diallo, 2006), l'article expose la méthode d'analyse des pratiques basée sur les savoirs locaux à partir d'enquêtes originales complétées par des suivis de troupeaux au pâturage; il présente les références obtenues et leur spécificité, discute de l'apport des savoirs locaux à l'intelligibilité des pratiques, du caractère actionnable des références produites et enfin de leur caractère générique. 


\section{Comment analyser les pratiques en prenant en compte les savoirs techniques locaux?}

\section{Élaboration de la démarche}

Les savoirs techniques locaux recouvrent de nombreux domaines. Avenier et Schmitt (2007) les distinguent des connaissances, qui sont par nature individuelles, à la fois produits de l'intériorisation des savoirs par le sujet et résultat de ses propres expériences cognitives.

Deux processus concomitants sont à l'œuvre dans l'élaboration d'un savoir technique : la distinction, division du réel en entités homogènes actionnables, et l'association, qui vise à relier les entités à des caractères, des valeurs. Un savoir dans un domaine se réfère à des savoirs plus élémentaires. Par exemple, le savoir sur les unités pastorales fait référence, entre autres, aux savoirs sur les sols, et le savoir sur les sols, aux savoirs et aux perceptions sur les couleurs, les textures, etc., ainsi de suite par régression successive jusqu'aux niveaux les plus élémentaires des savoirs, acquis dès le plus jeune âge par la fonction cognitive de distinction (Cyrulnik, 1995).

Ainsi, un savoir dans un domaine est relativement structuré. Il repose sur un système de classification (entités actionnables), chaque entité s'énonçant en référence à des variables de caractérisation (description, fonctionnalité, risque) et à des indicateurs d'état. Nos travaux sur le savoir portant sur les sols, les semences, la fumure organique, les animaux de trait, les saisons des pluies nous ont permis de le vérifier à chaque fois. Cette structuration n'est pour autant ni figée ni homogène. D'un individu à l'autre, les savoirs sont variables et inégalement partagés, ce qui permet de distinguer un fonds commun de connaissances et des connaissances spécialisées, voire monopolisées (Darré et al., 2004). D'une époque à l'autre, les savoirs évoluent et se transforment en fonction des apports de connaissances extérieures et de l'évolution de la situation locale. L'expression des savoirs dépend du positionnement du sujet dans l'environnement social, économique et physique. L'introduction de nouveaux savoirs dans un groupe social provoque une confrontation avec les savoirs locaux (Scoonnes et Thompson, 1999), mettant en jeu une mécanique de traductions complexes (Akrich et al., 2006) au sein des réseaux d'acteurs locaux, pour construire, par l'échange et le débat, de nouveaux savoirs et de nouvelles pratiques.

Savoirs et pratiques forment un tout en interaction. Le savoir technique local constitue ainsi une théorie de l'action, une façon de concevoir les choses, l'intelligence de ce qui est et de programmation du faire. Les règles de gestion se situent donc à la frontière entre savoirs et pratiques.

Considérant ces éléments théoriques, nous proposons une démarche d'analyse des pratiques en deux phases. La première phase est consacrée à la caractérisation du savoir technique local dans le domaine considéré : identification des entités composant le système de classification ; caractérisation des variables de description, de fonctionnalité et de risque liées à ces entités ; identification des indicateurs utilisés par les praticiens pour évaluer l'état des variables; identification des règles de gestion se référant aux entités du système de classification. La seconde phase est consacrée à l'étude des pratiques par des observations directes en se référant aux systèmes de classification locaux identifiés à la première étape.

\section{Application de la démarche à l'étude présentée}

Cette démarche a été appliquée pour l'étude des pratiques de conduite des troupeaux au pâturage sur un échantillon de dix éleveurs des villages de Koumbia et de Waly, suivis de juin 2005 à juillet 2006.

\section{Caractérisation des savoirs techniques locaux dans deux domaines}

En rapport avec la finalité de ce travail visant l'élaboration par les acteurs locaux d'une convention précisant les règles de conduite des troupeaux au pâturage dans le temps et dans l'espace, nous avons choisi d'étudier spécifiquement les savoirs techniques des éleveurs relatifs au calendrier pastoral et aux espaces pastoraux, afin d'identifier les règles de conduite au cours de l'année dans les territoires exploités par les éleveurs. L'analyse s'est déroulée en trois étapes :

1. enquêtes exploratoires pour identifier les entités des systèmes de classification (périodes, unités pastorales), les variables de caractérisation, les indicateurs et les règles ;

2. enquêtes individuelles approfondies chez les dix éleveurs, dans le but de caractériser la diversité des connaissances individuelles à partir des éléments recueillis lors de la première étape; construction du fonds commun de connaissances et repérage des variantes;

3. restitution des résultats à la communauté des éleveurs pour compléter et valider les représentations du savoir technique local dans le domaine considéré et ses variantes ; réalisation d'investigations complémentaires (cartes à dire d'acteurs, etc.).

Pour faciliter la lecture, les termes fulfulde du texte ont été regroupés dans l'encadré 2.

\section{Caractérisation des pratiques de conduite au pâturage}

L'étude des pratiques a été réalisée en référence aux entités du savoir technique local (périodes et unités pastorales). Les troupeaux ont fait l'objet d'un suivi diurne au pâturage, une fois pour chacune des périodes du calendrier pastoral. Le long du parcours, la prise de 


\section{Encadré 2. Lexique des termes fulfulde utilisés dans le texte}

Le lexique suivant répertorie les termes fulfulde (langue peule) utilisés dans cet article. Ils sont couramment employés par les populations peules dans cette partie de l'Ouest burkinabe. À Koumbia-Waly, les éleveurs peuls représentent $9 \%$ des unités de production (UP) du village. On peut aussi noter la présence de nombreux bergers peuls salariés, responsables de la conduite des troupeaux des agro-éleveurs bwaba et mossi (7\% des UP). Les autres acteurs, détenteurs de troupeaux de petite taille et de bœufs de trait, ont tendance à s'approprier certains termes fulfulde pour la gestion de leurs troupeaux. Le phénomène le plus répandu chez les agriculteurs est l'utilisation des termes peuls dans les noms donnés aux animaux.

Ce vocabulaire est encore peu fréquent dans les études agropastorales réalisées dans la région. Certains chercheurs biotechniciens mentionnent des éléments du lexique dans leurs études locales sur le pastoralisme (Kagoné 2000 ; Kiéma 2007). Mais, en général, ces termes disparaissent du vocabulaire des chercheurs et des développeurs lorsque le travail évolue vers une phase d'intervention. NB : dans le texte, les termes peuls seront toujours utilisés au singulier pour faciliter la lecture. Noter qu'en début de mot le $c$ se prononce $[t c h]$; le $j$ se prononce $[d j]$.

\begin{tabular}{|c|c|c|}
\hline Singulier & Pluriel & Définition \\
\hline bolaawo & - & pâturage de zone inondable (savane arborée, arbustive) \\
\hline bomboru & - & pâturage de zone inondable (savane arborée, forêt claire) \\
\hline burtol (gursol) & burti (gursi) & piste à bétail \\
\hline ceedu & ceduji & saison sèche et chaude \\
\hline cofol & coofi & pâturage de bas-fonds (formations ripicoles) \\
\hline dabbude & dabbule & saison sèche et froide \\
\hline ferlo & perde & pâturage de colline (savane arborée, arbustive) \\
\hline fukkaawo & fukkaaje & pâturage de colline (savane arbustive, herbeuse) sur cuirasse affleurante \\
\hline gadaawe & gadaaji & animal de queue \\
\hline gataaje & gate & début de saison des pluies \\
\hline gesa & gese & champ cultivé \\
\hline guyfan (guumal) & guube & relique arborée ou arbustive dense \\
\hline hoore & horeeji & animal de tête \\
\hline hudo & - & herbe \\
\hline ladde & laddeedji & brousse \\
\hline maanje & - & pâture matinale \\
\hline mbundu & mbulli & puits \\
\hline nabbere & nabbe & petite mare de plaine inondable \\
\hline ndungu & ndunguuji & saison des pluies (hivernage) \\
\hline nyayle & - & résidus de cultures pâturables sur pieds \\
\hline parkoru & parkoji & lentille d'eau \\
\hline seeno & ceene & pâturage de plaine sèche sablonneuse (savane arborée, arbustive) \\
\hline seraawe & seraaji & animal de flanc \\
\hline soggude & - & pâture nocturne \\
\hline soynere (dancere) & coyde (dance) & jachère \\
\hline yaamde & yaamle & fin de saison pluvieuse, début de récolte \\
\hline yayre & - & étendue d'eau dans les bas-fonds \\
\hline
\end{tabular}

données était déclenchée par l'entrée dans une nouvelle unité pastorale, définie selon la nomenclature peule : relevé de position (latitude/longitude, heure), complété par des observations continues sur le troupeau et son environnement (activité du troupeau, caractéristiques du milieu). Les parcours ont ainsi été caractérisés par la durée, la distance parcourue, la vitesse du troupeau, le nombre de stations de broutage, la forme du circuit, la durée du repos, du broutage, de l'abreuvement, et la contribution spécifique des faciès au broutage. Cette dernière donnée a été plus spécifiquement analysée dans cet article pour préciser les chaînes annuelles de pâturage définies par les couples "période du calendrier $x$ types de pâturages fréquentés » (Kagoné, 2000), leur diversité, l'importance relative des différentes unités pastorales et les possibilités d'ajustements.

Comparativement aux méthodes de suivi généralement utilisées pour ce type d'étude (Ickowicz et Mbaye,
2001 ; Botoni, 2003), celle que nous proposons présente des particularités permettant d'accéder à des données originales sur les pratiques de conduite. Dans les études classiques, les faciès de végétation sont définis a priori par les scientifiques (Aubreville, 1957). Les périodes de suivi tiennent compte des saisonnalités, mais ignorent souvent des moments critiques reconnus par les éleveurs. Les relevés au pâturage (position, prise alimentaire) sont effectués à intervalles réguliers et fixés au départ. Ce type de démarche débouche en général sur une description externe de la conduite du troupeau et de l'exploitation des milieux, complétée par une évaluation quantitative de la potentialité des espaces (capacité de charge...) et des effets du pâturage sur les ressources. En revanche, il ne permet pas de saisir ce que les périodes et les pâturages représentent pour les praticiens, d'analyser finement les changements de pratiques d'un milieu à l'autre, de comprendre les logiques d'enchaînement, ni de produire des 
Tableau 1. Savoir technique local relatif au calendrier pastoral.

\begin{tabular}{|c|c|c|c|c|c|c|c|}
\hline \multicolumn{2}{|c|}{ Entités du calendrier pastoral } & \multirow{2}{*}{$\begin{array}{c}\text { Gataaje } \\
\begin{array}{c}\text { Début de saison } \\
\text { des pluies } \\
\text { (mai-juin) }\end{array}\end{array}$} & \multirow{2}{*}{$\begin{array}{c}\text { Ndungu } \\
\text { Saison des } \\
\text { pluies } \\
\text { (juil.-sep.) }\end{array}$} & \multirow{2}{*}{$\begin{array}{l}\text { Yaamde } \\
\text { Début des } \\
\text { récoltes } \\
\text { (octobre) }\end{array}$} & \multirow{2}{*}{ 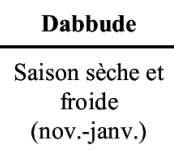 } & \multirow{2}{*}{$\begin{array}{c}\text { Ceedu } \\
\text { Saison sèche et } \\
\text { chaude } \\
\text { (fév.-avril) }\end{array}$} & \multirow{4}{*}{ Indicateurs } \\
\hline \multirow{3}{*}{$\begin{array}{l}\text { Variable de } \\
\text { description }\end{array}$} & Français & & & & & & \\
\hline & Arabe (Tchad) & richach & karif & davat & chité & seif & \\
\hline & $\begin{array}{l}\text { Fulfulde } \\
\text { (Cameroun) }\end{array}$ & seeto & ndungu & yaamde & dabbude & ceedu & \\
\hline \multirow{2}{*}{$\begin{array}{l}\text { Variable de } \\
\text { fonctionna- } \\
\text { lité }\end{array}$} & $\begin{array}{l}\text { Disponibilité et } \\
\text { accessibilité à } \\
\text { l'eau }\end{array}$ & & & & & f & $\begin{array}{l}\text { Aspect des eaux } \\
\text { de surface } \\
\text { (sain ou pourri) }\end{array}$ \\
\hline & $\begin{array}{l}\text { Disponibilité et } \\
\text { qualité fourragère }\end{array}$ & & & f & f & 手 & $\begin{array}{l}\text { Directs : sur les } \\
\text { unités pastorales } \\
\text { (abondance...) } \\
\text { Indirects : creux } \\
\text { flanc droit des } \\
\text { bovins }\end{array}$ \\
\hline $\begin{array}{l}\text { Variable de } \\
\text { risque }\end{array}$ & $\begin{array}{l}\text { Risque de conflit } \\
\text { avec les } \\
\text { agriculteurs }\end{array}$ & & & f & f & f & $\begin{array}{l}\text { État des semis } \\
\text { et implantation } \\
\text { des cultures }\end{array}$ \\
\hline \multicolumn{2}{|c|}{$\begin{array}{l}\text { Règles de conduite du troupeau au } \\
\text { pâturage selon les périodes }\end{array}$} & $\begin{array}{l}\text { Rechercher les } \\
\text { zones de } \\
\text { premières } \\
\text { pluies et de } \\
\text { repousse de } \\
\text { l'herbe }\end{array}$ & $\begin{array}{l}\text { Reconstituer les } \\
\text { réserves } \\
\text { corporelles des } \\
\text { bovins ; } \\
\text { éviter les } \\
\text { dégâts sur les } \\
\text { cultures }\end{array}$ & $\begin{array}{l}\text { Éviter les } \\
\text { dégâts sur les } \\
\text { récoltes, } \\
\text { négociation des } \\
\text { « contrats » de } \\
\text { vaine pâture }\end{array}$ & $\begin{array}{l}\text { Soutenir la } \\
\text { reconstitution } \\
\text { des réserves et } \\
\text { les femelles } \\
\text { suitées ; } \\
\text { " course » à la } \\
\text { vaine pâture }\end{array}$ & $\begin{array}{l}\text { Faire boire le } \\
\text { troupeau et } \\
\text { trouver de quoi } \\
\text { pâturer ; } \\
\text { si nécessité, } \\
\text { départ en } \\
\text { transhumance }\end{array}$ & \\
\hline
\end{tabular}

références ayant du sens pour les acteurs de terrain et dotés d'un caractère actionnable.

\section{Savoirs techniques des éleveurs et pratiques de conduite aux pâturages}

\section{Caractérisation des savoirs techniques des éleveurs relatifs au temps et à l'espace}

\section{Gestion du troupeau dans le temps : le calendrier pastoral}

Les éleveurs découpent l'année en cinq périodes, auxquelles ils se réfèrent toujours lorsqu'ils parlent de la conduite de leur troupeau (Tab. 1) :

1. gataaje : début de la saison des pluies

2. ndungu : saison des pluies (hivernage)

3. yaamde: début des récoltes, fin de l'hivernage

4. dabbude: saison sèche et froide

5. ceedu : saison sèche et chaude.

Ce découpage se retrouve ailleurs en Afrique subsaharienne, comme l'indiquent les déclinaisons des noms des périodes en fulfulde (Cameroun) et en arabe (Tchad).
Chaque période est caractérisée par deux variables de fonctionnalité (disponibilité et accessibilité à l'eau, disponibilité et qualité fourragère) et une variable de risque (potentialité de conflits avec les agriculteurs). Le contexte sanitaire a rarement été cité comme élément de caractérisation.

Les éleveurs sont très sensibles à l'évolution de la disponibilité et de l'accessibilité à l'eau. Du début de la saison des pluies au début des récoltes, les eaux de surface sont abondantes et saines, puis elles se raréfient de la saison sèche et froide à la saison sèche et chaude. Les éleveurs distinguent différents types de point d'abreuvement selon leur position sur la toposéquence et selon les périodes. Sur les collines, on trouve en hivernage des lentilles d'eau (parkoru) qui s'assèchent vite. Les plaines inondables présentent de-ci de-là des petites mares (mbundu) et les bas-fonds des étendues d'eau persistantes (yayre). Enfin dans les zones de culture, les éleveurs creusent des puisards en saison sèche (mbulli). Pour apprécier la qualité de l'eau, ils ont des indicateurs directs relatifs à son aspect, son odeur (sain, pourri) et des indicateurs indirects sur l'animal (une eau de mauvaise qualité donne un pelage terne, des poils hérissés). Les points d'abreuvement changent à chaque période, ce qui oriente les itinéraires de pâturage. 
La disponibilité et la qualité fourragères des espaces pâturables oscillent entre des périodes d'abondance (hivernage, saison sèche et froide), des intersaisons difficiles (début de la saison des pluies, début des récoltes) et une période de disette durant la saison sèche et chaude. Les pâturages ne présentent donc pas le même intérêt pour les éleveurs et leurs animaux au fil de l'année. L'intérêt d'un pâturage est évalué par des observations directes sur la ressource : abondance et persistance de la biomasse fourragère, facteurs de risques pouvant le dévaluer, parasites, pathologies, plantes toxiques..., mais aussi par des indicateurs indirects observés sur l'animal, tels que la concavité du flanc gauche au retour du pâturage (plus le flanc est concave, plus la prise alimentaire est jugée insuffisante), l'aspect du pelage (s'il est lisse et brillant, cela indique la fréquentation répétée de bons pâturages), la valeur alimentaire ou «force » du pâturage (capacité à faire du lait, du gras ou bien du muscle).

Dans les zones agropastorales, le risque de conflit est un élément d'appréciation de la valeur pastorale d'un espace. Les principales périodes de conflits (résultant de dégâts des troupeaux sur les champs non récoltés, de contestation du droit de vaine pâture) se situent au début des récoltes, au début de la saison sèche et froide et, dans une moindre mesure, durant l'hivernage (installation des champs sur les pistes à bétail et au ras des campements). Pour prévenir les risques de conflit, les éleveurs observent l'état d'implantation des cultures. Ces observations portent non seulement sur la campagne agricole en cours, mais aussi et surtout sur l'évolution interannuelle de l'emprise des champs.

Chaque période se caractérise par des règles spécifiques de conduite du troupeau au pâturage. En début d'hivernage, les animaux affaiblis par la rigueur de la saison sèche et chaude commencent à reconstituer leurs réserves corporelles avec la reprise progressive du couvert herbacé et la feuillaison des ligneux appréciés. Les éleveurs recherchent les zones de premières pluies et, lorsque l'hivernage tarde à s'installer, certains quittent temporairement le terroir pour des zones mieux arrosées indiquées par des pairs. Dès que l'herbe recommence à pousser, la crise fourragère prend fin, les difficultés changent de nature. C'est la reprise de la surveillance pour prévenir les dégâts sur les premiers semis.

Durant l'hivernage, la ressource fourragère est abondante, mais les risques de conflit sont élevés. Les éleveurs recherchent les espaces avec peu de champs et font en sorte que les animaux profitent le plus possible d'une biomasse herbacée spontanée et abondante.

L'imminence des récoltes accroît la gravité des dégâts aux cultures. Durant cette période, les éleveurs replient les troupeaux sur les collines, bien que la ressource alimentaire y soit médiocre, les herbacées annuelles ayant bouclé leur cycle. Les propriétaires de troupeaux négocient avec les agriculteurs des droits de pâture des résidus, préparant ainsi la saison sèche et froide.

Durant la saison sèche et froide - moment de la vaine pâture -, la recherche des zones où abondent les résidus agricoles (nyayle) pour soutenir la reconstitution des réserves corporelles et la lactation des vaches suitées conduit certains éleveurs à des déplacements de faible amplitude et de courte durée. La pratique du pâturage nocturne, abandonnée depuis le début de l'hivernage, reprend. Avec l'assèchement des points d'eau de surface, l'abreuvement s'effectue aux puisards creusés dans les bas-fonds et les champs.

Durant la saison sèche et chaude, il s'agit de limiter l'amaigrissement des animaux. La crise fourragère est atténuée par l'exploitation des ligneux et l'apport parcimonieux du tourteau et de la graine de coton aux femelles gestantes, aux femelles suitées et aux bovins de trait (Blanchard, 2005). Les puisards sont quelquefois asséchés et l'abreuvement s'effectue au forage par rotation. Si l'abreuvement devient trop problématique, l'éleveur part en transhumance vers des zones plus propices.

\section{La répartition spatiale des ressources et leur exploitation : unités pastorales et chaînes de pâturage}

À la notion de temps se superpose celle de l'espace pour former ainsi un tout, difficilement dissociable quand on cherche à comprendre l'organisation spatiotemporelle de la conduite des troupeaux au pâturage.

Ladde (brousse en fulfulde) est le terme générique utilisé par les éleveurs pour parler de l'endroit où les troupeaux sont conduits quotidiennement pour assurer leur ration alimentaire et leur abreuvement. Ladde, zone sans culture, sans habitation et où l'on trouve de l'herbe, des fourrages ligneux et de l'eau, représente en réalité un vaste ensemble, dans lequel les éleveurs distinguent des unités pastorales plus fines. Ces unités se différencient selon la toposéquence (Fig. 1).

Le tableau 2 présente les huit principales unités pastorales identifiées, parmi lesquelles six relèvent des parcours naturels et deux des zones ayant subi l'action de l'homme à travers leur mise en culture passée ou actuelle. Les éleveurs les caractérisent par des variables de description (types de sol et toposéquence, espèces herbacées et ligneuses, eau), des variables fonctionnelles (intérêt pastoral) et des variables de risque. Un travail similaire réalisé avec les éleveurs de Kourouma, village situé à $150 \mathrm{~km}$ au nord-ouest de Koumbia (Diallo, 2007), a permis de mettre en évidence une nomenclature des unités pastorales construite selon la même logique. Les deux systèmes de classification comportent une majorité d'unités identiques (ferlo, fukkaawo, seeno, cofol, soynere, gesa) et quelques unités spécifiques à chaque territoire villageois. Ce résultat illustre la notion de fonds commun de connaissances partagées par les éleveurs peuls de l'Ouest burkinabé et 


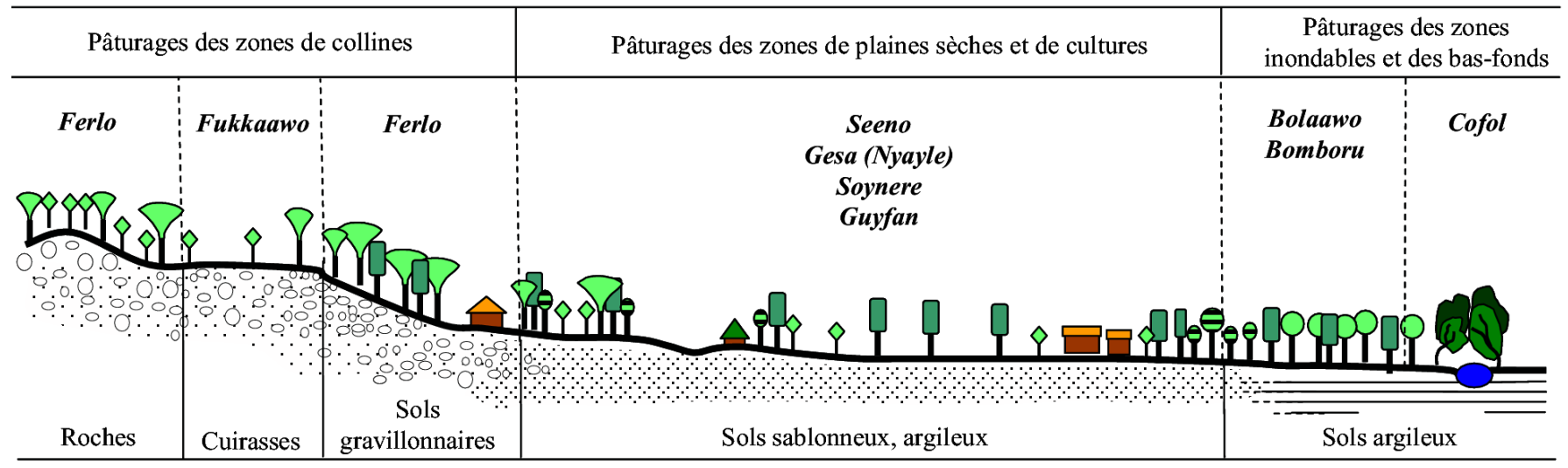

Fig. 1. Répartition des unités pastorales suivant la toposéquence.

de variantes locales liées au contexte de chaque zone et marquant des ajustements des modes de conduite des troupeaux.

L'usage de ces unités varie en fonction des périodes au regard de leur accessibilité et des ressources qu'elles offrent pour constituer la ration des troupeaux qui les parcourent. La séance de restitution à été l'occasion de traiter avec les éleveurs de l'exploitation chronologique aux différentes périodes de cette diversité d'unités pastorales. Ce travail a permis de dégager ce que nous avons appelé la chaîne annuelle théorique de pâturage, qui représente les unités pastorales intéressantes où, en général, les éleveurs conduiraient prioritairement leurs troupeaux au fil des périodes. Elle a été validée par la communauté des éleveurs (Tab. 3).

Au début de la saison des pluies (gataaje), le broutage s'effectue principalement là où l'herbe repousse vite, dans les bas-fonds ( $c o f o l)$ et sur les zones en attente d'une mise en culture (soynere et gesa). Durant l'hivernage (ndungu), les jachères (soynere) et les unités de collines (ferlo, fukkaawo) gagnent de l'intérêt; sont alors exclues des parcours les zones cultivées (gesa). Au début des récoltes (yaamde), les pâturages se font principalement le long des bas-fonds ( $c o f o l)$, secondairement sur les jachères (soynere), qui perdent de l'intérêt par rapport à la période précédente, et surtout sur les premiers résidus agricoles (gesa). La nuit, certains éleveurs installent temporairement le parc à bétail sur les collines (ferlo), à l'écart des champs en attente de récolte. Durant la saison sèche et froide (dabbude), la récolte est finie, la grande majorité du temps de broutage se déroule dans le domaine agricole, sur les résidus (nyayle). Tout au long de la saison sèche et chaude $(c e e d u)$, les troupeaux parcourent les parties du domaine agricole (gesa et soynere) épargnées par le feu, les pâturages des zones inondables (cofol, bolaawo, bomboru) pour les repousses des herbacées vivaces et, en fin de circuit, le ferlo pour l'émondage des ligneux fourragers.

L'espace pastoral comporte aussi des zones de surfaces limitées mais très importantes pour la circulation et l'alimentation du bétail. Les burtol (pistes à bétail) permettent de rallier les points d'eau, les pâturages et les parcs de nuit. Les guyfan, reliques de savanes arborées et arbustives denses, forment un lacis d'interstices entre les champs servant de passage en hivernage et de pâture durant la saison sèche et chaude.

\section{Variabilité des pratiques de pâturage et diversité des conflits agropastoraux}

\section{Variabilité des pratiques de pâturage}

La chaîne de pâturage théorique met en évidence les principes généraux de la conduite du troupeau selon les périodes. Cependant, lors de la restitution des travaux, les éleveurs ont rappelé que ce schéma théorique pouvait varier dans son application réelle suivant un certain nombre d'éléments (l'individu, sa position sur le territoire, sa position sociale, les années, la taille du troupeau...). Pour mieux interpréter cette variabilité, nous avons analysé les pratiques de conduite réelles de deux éleveurs (E1 et E2) et comparé leurs chaînes de pâturage réelles avec la chaîne de pâturage théorique. E1 est un agro-éleveur mossi propriétaire d'un troupeau de 30 à 40 bovins, constitué depuis 20 ans et gardé par son fils dans un objectif d'épargne des revenus du coton. E2 est un éleveur peul propriétaire d'un troupeau d'une soixantaine de têtes, gardé par son fils. E2 a une longue expérience de l'élevage. Il exploite son troupeau par la vente du lait et des animaux.

La figure 2 représente, pour les deux éleveurs, les contributions des unités de pâturage au temps de broutage selon les périodes et met en correspondance ces mesures avec les éléments de la chaîne de pâturage théorique. L'analyse du graphe indique tantôt une adéquation, tantôt des écarts entre la théorie et la pratique, révélant ainsi un double aspect des modes de conduite des troupeaux au pâturage : leur régularité (lorsque la pratique correspond à la description théorique) et leur flexibilité face à l'aléa (dans le cas contraire). 


\begin{tabular}{|c|c|c|c|c|c|c|c|c|c|}
\hline \multirow{2}{*}{ 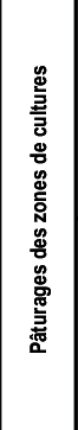 } & 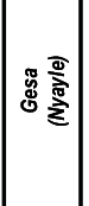 & 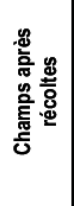 & $\begin{array}{l}\frac{20}{02} \\
\frac{2}{0} \\
\frac{0}{8} \\
0\end{array}$ & 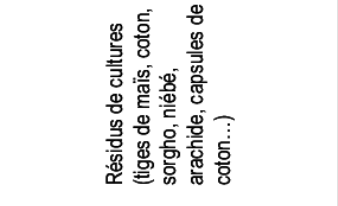 & 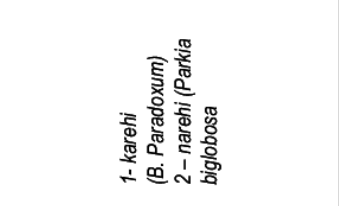 & $\begin{array}{l}\text { 唁 } \\
\text { 言 } \\
\text { हn }\end{array}$ & 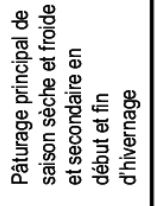 & 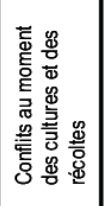 & 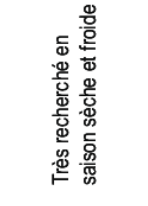 \\
\hline & 产 & 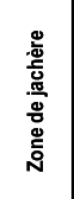 & 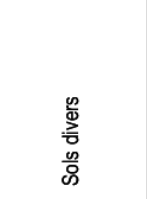 & 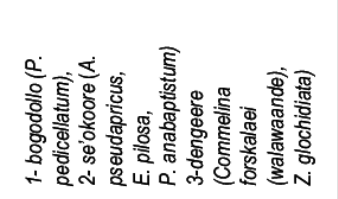 & 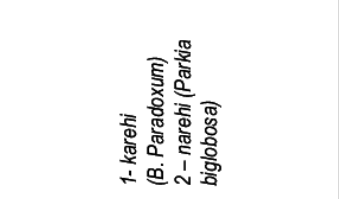 & 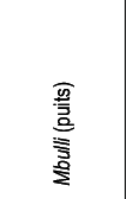 & 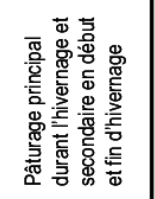 & 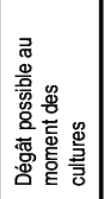 & 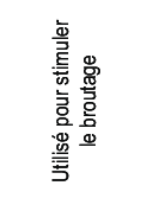 \\
\hline \multirow{3}{*}{ 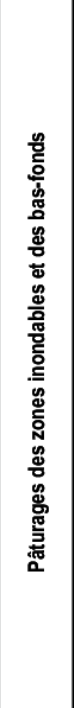 } & $\begin{array}{l}\text { 롱 } \\
\text { हूँ } \\
\text { है }\end{array}$ & 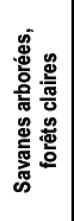 & 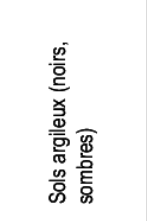 & 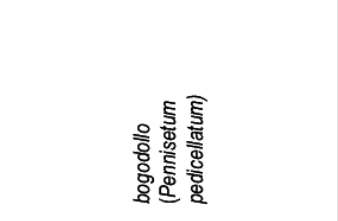 & 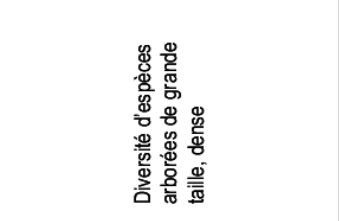 & 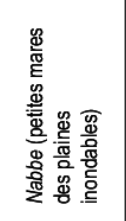 & 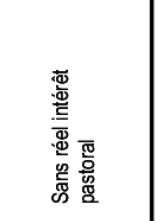 & 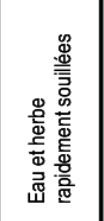 & 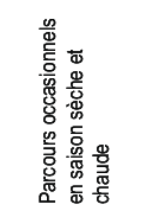 \\
\hline & 愛 & 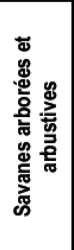 & 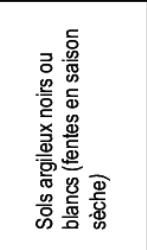 & 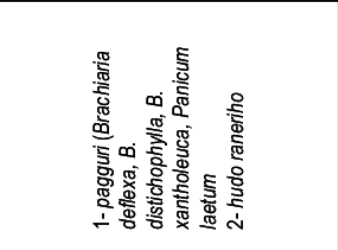 & 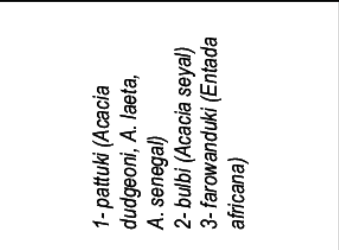 & 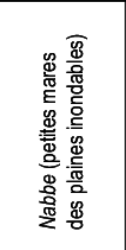 & 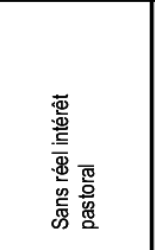 & 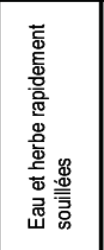 & 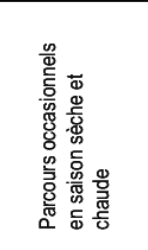 \\
\hline & 홍 & 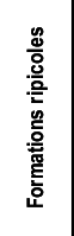 & 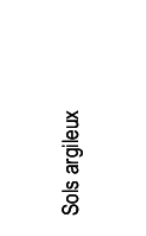 & 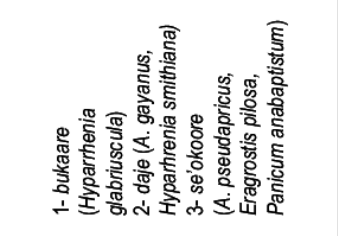 & 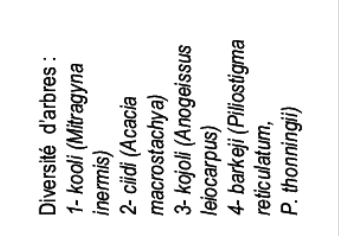 & 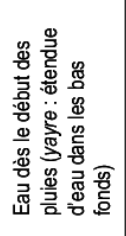 & 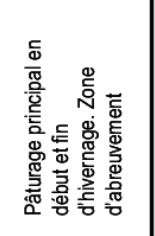 & 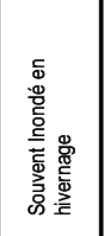 & 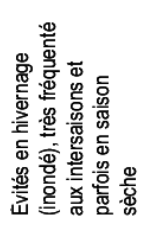 \\
\hline 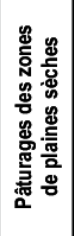 & 怘 & 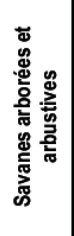 & 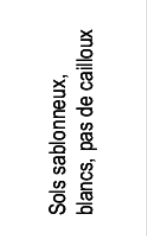 & 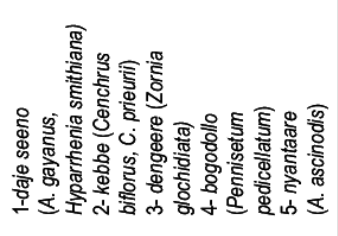 & 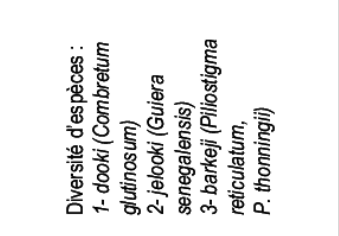 & 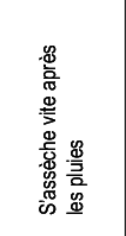 & 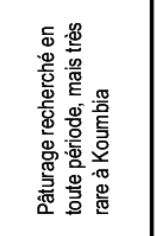 & & 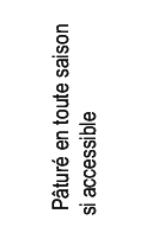 \\
\hline \multirow{2}{*}{ 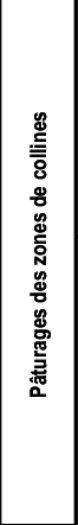 } & $\begin{array}{l}\text { 宽 } \\
\text { 竎 } \\
\text { 竎 }\end{array}$ & 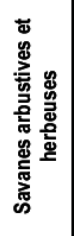 & 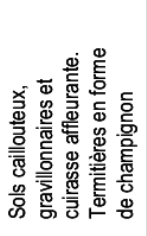 & 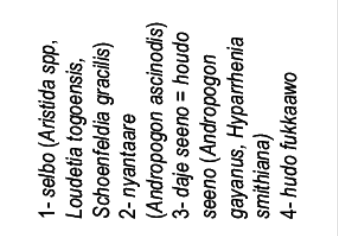 & 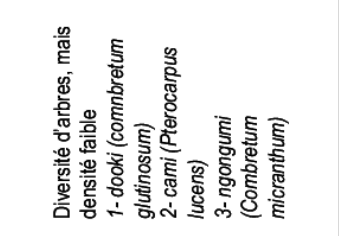 & 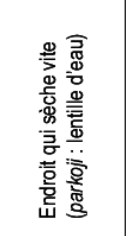 & 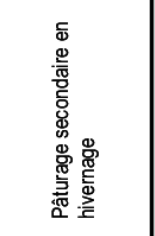 & 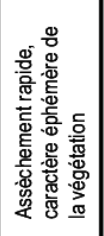 & 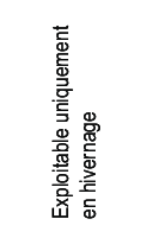 \\
\hline & 暹 & 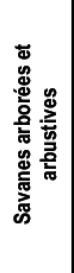 & 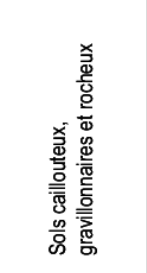 & 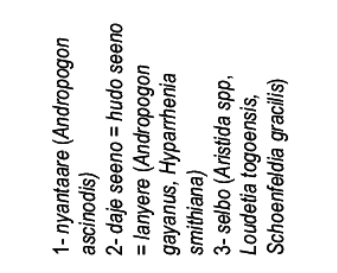 & 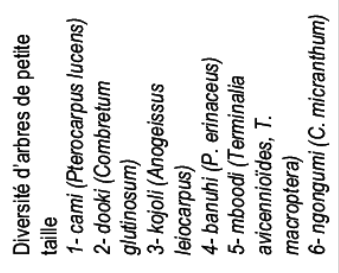 & 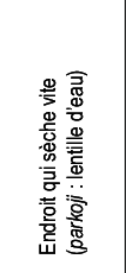 & 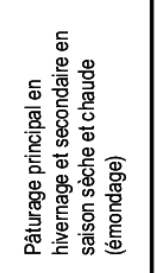 & 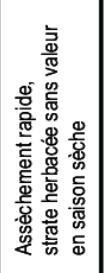 & 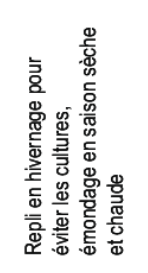 \\
\hline \multirow{2}{*}{\multicolumn{3}{|c|}{ 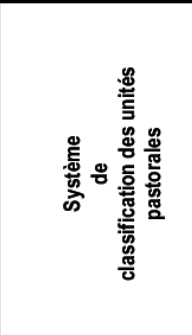 }} & 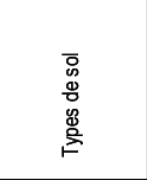 & 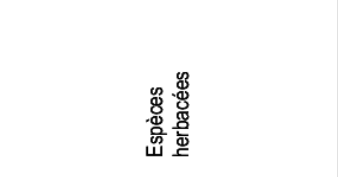 & 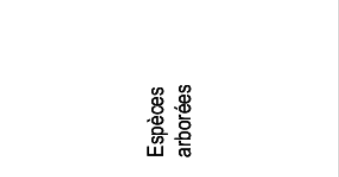 & 芭 & \multirow[b]{2}{*}{$>$} & \multirow{2}{*}{ 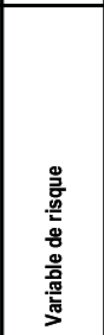 } & \multirow{2}{*}{ 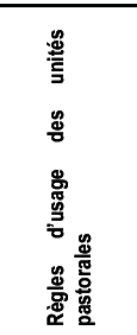 } \\
\hline & & & \multicolumn{4}{|c|}{ 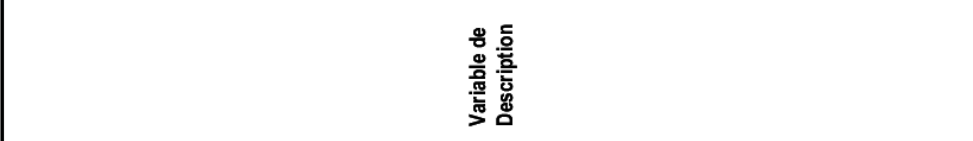 } & & & \\
\hline
\end{tabular}


Tableau 3. Chaîne de pâturage théorique.

\begin{tabular}{|c|c|c|c|c|c|c|c|c|}
\hline \multirow{3}{*}{ Unités pastorales } & \multicolumn{2}{|c|}{$\begin{array}{l}\text { Pâturages des zones de } \\
\text { collines }\end{array}$} & \multirow{2}{*}{$\begin{array}{c}\text { Pâturages } \\
\text { des zones de } \\
\text { plaines } \\
\text { sèches } \\
\text { Seeno } \\
\end{array}$} & \multicolumn{3}{|c|}{$\begin{array}{l}\text { Pâturages des zones inondables et des bas- } \\
\text { fonds }\end{array}$} & \multicolumn{2}{|c|}{$\begin{array}{l}\text { Pâturages des zones de } \\
\text { cultures }\end{array}$} \\
\hline & Ferlo & Fukkaawo & & Cofol & Bolaawo & Bomboru & Soynere & Gesa \\
\hline & $\begin{array}{l}\text { Savanes } \\
\text { arborées et } \\
\text { arbustives }\end{array}$ & $\begin{array}{l}\text { Savanes } \\
\text { arbustives et } \\
\text { herbeuses }\end{array}$ & $\begin{array}{l}\text { Savanes } \\
\text { arborées et } \\
\text { arbustives }\end{array}$ & $\begin{array}{l}\text { Formations } \\
\text { ripicoles }\end{array}$ & $\begin{array}{l}\text { Savanes } \\
\text { arborées et } \\
\text { arbustives }\end{array}$ & $\begin{array}{c}\text { Savanes } \\
\text { arborées, } \\
\text { forêts claires }\end{array}$ & $\begin{array}{l}\text { Zone de } \\
\text { jachère }\end{array}$ & $\begin{array}{l}\text { Champs } \\
\text { après } \\
\text { récoltes }\end{array}$ \\
\hline $\begin{array}{l}\text { Gataaje } \\
\text { Début de saison } \\
\text { des pluies } \\
\text { (mai-juin) }\end{array}$ & + & - & ++ & +++ & - & - & ++ & ++ \\
\hline $\begin{array}{l}\text { Ndungu } \\
\text { Saison des pluies } \\
\text { (juil.-sep.) }\end{array}$ & +++ & ++ & ++ & + & - & - & +++ & - \\
\hline $\begin{array}{l}\text { Yaamde } \\
\text { Début des récoltes } \\
\text { (octobre) }\end{array}$ & ++ & + & ++ & +++ & + & - & ++ & + \\
\hline $\begin{array}{l}\text { Dabbude } \\
\text { Saison sèche } \\
\text { et froide } \\
\text { (nov.-janv.) }\end{array}$ & + & - & ++ & + & - & - & - & +++ \\
\hline $\begin{array}{l}\text { Ceedu } \\
\text { Saison sèche } \\
\text { et chaude } \\
\text { (fév.-avril) }\end{array}$ & ++ & - & ++ & + & + & + & + & ++ \\
\hline
\end{tabular}

Légende : +++ pâturage principal ; ++ pâturage secondaire ; + pâturage ayant peu d'intérêt ; - pâturage sans intérêt ou impossible

Souvent, la situation observée ne correspond pas à la description de la pratique habituelle (exemple : absence de pâturage dans le ferlo durant l'hivernage). Ces situations révèlent l'existence de pratiques circonstancielles opérées soit par opportunité, soit sous la contrainte. Le tableau 4 indique les causes des ajustements observés chez E1 et E2. Les ajustements opérés en fonction des situations de chaque éleveur sont à la base de toute la diversité que l'on peut observer en matière de pratique de pâturage. Ces ajustements s'opèrent aussi au niveau des rythmes de pâturage imposés aux troupeaux sur les circuits de pâture, qui ont des incidences sur les performances des troupeaux.

Ainsi, les chaînes de pâturage et les modes de conduite sont ajustés selon la situation individuelle, selon l'évolution de l'occupation de l'espace au fil des périodes et selon les scénarios pluviométriques annuels. Dans un environnement caractérisé par de multiples incertitudes et aléas, cette flexibilité à caractère fonctionnel (Dedieu et al., 2008) constitue sans aucun doute une garantie de durabilité pour l'élevage et devra être préservée, notamment en cherchant à sécuriser la mobilité des troupeaux sur le territoire.

\section{Conflits agropastoraux et stratégies d'adaptation}

Selon les déclarations des acteurs de terrain et de l'administration, la majorité des conflits surviennent entre le début des récoltes et la fin de l'évacuation des productions agricoles des champs (entre yaamde et dabbude). Durant cette période, les conflits proviennent des dégâts des troupeaux sur des champs non récoltés (maïs, coton) ou sur les récoltes gardées au champ (coton). Pour les éviter, certains éleveurs délocalisent le parc de nuit sur les collines (ferlo) au début des récoltes (yaamde), de façon à écarter le troupeau de la zone agricole. D'autres conflits découlent de la pâture nocturne (soggude) des résidus de culture effectuée sans l'accord du propriétaire du champ, spoliant ce dernier d'une ressource qu'il réservait pour ses propres animaux. Pour prévenir ces conflits, les éleveurs avisés négocient des droits de pâturage de résidus au démarrage des récoltes. Aujourd'hui, les résidus de culture sont de plus en plus convoités et le droit de vaine pâture est remis en question.

Durant l'hivernage, la situation s'inverse et l'éleveur passe du statut d'accusé à celui de plaignant. L'emprise agricole rend très problématique la mobilité du bétail sur la zone cultivée qu'il faut traverser chaque jour pour atteindre les points d'eau, les jachères (soynere) et les parcours de colline (ferlo, fukkaawo). Les difficultés sont accrues par les installations des champs au ras des campements, des points d'eau et sur les pistes à bétail (burtol), forçant quelquefois les détenteurs de grands troupeaux à quitter le territoire. Durant cette période, pour mieux canaliser les troupeaux dans les interstices non cultivés (guyfan), les éleveurs ont plusieurs stratégies : ils peuvent jouer sur l'éthologie des individus pour encadrer le troupeau par les animaux meneurs (hoore) et accorder plus d'attention aux animaux de flanc (seraawe) et de queue (gadaawe); les bergers de petits troupeaux peuvent s'associer pour garder à deux; les grands troupeaux sont divisés en deux lots. 


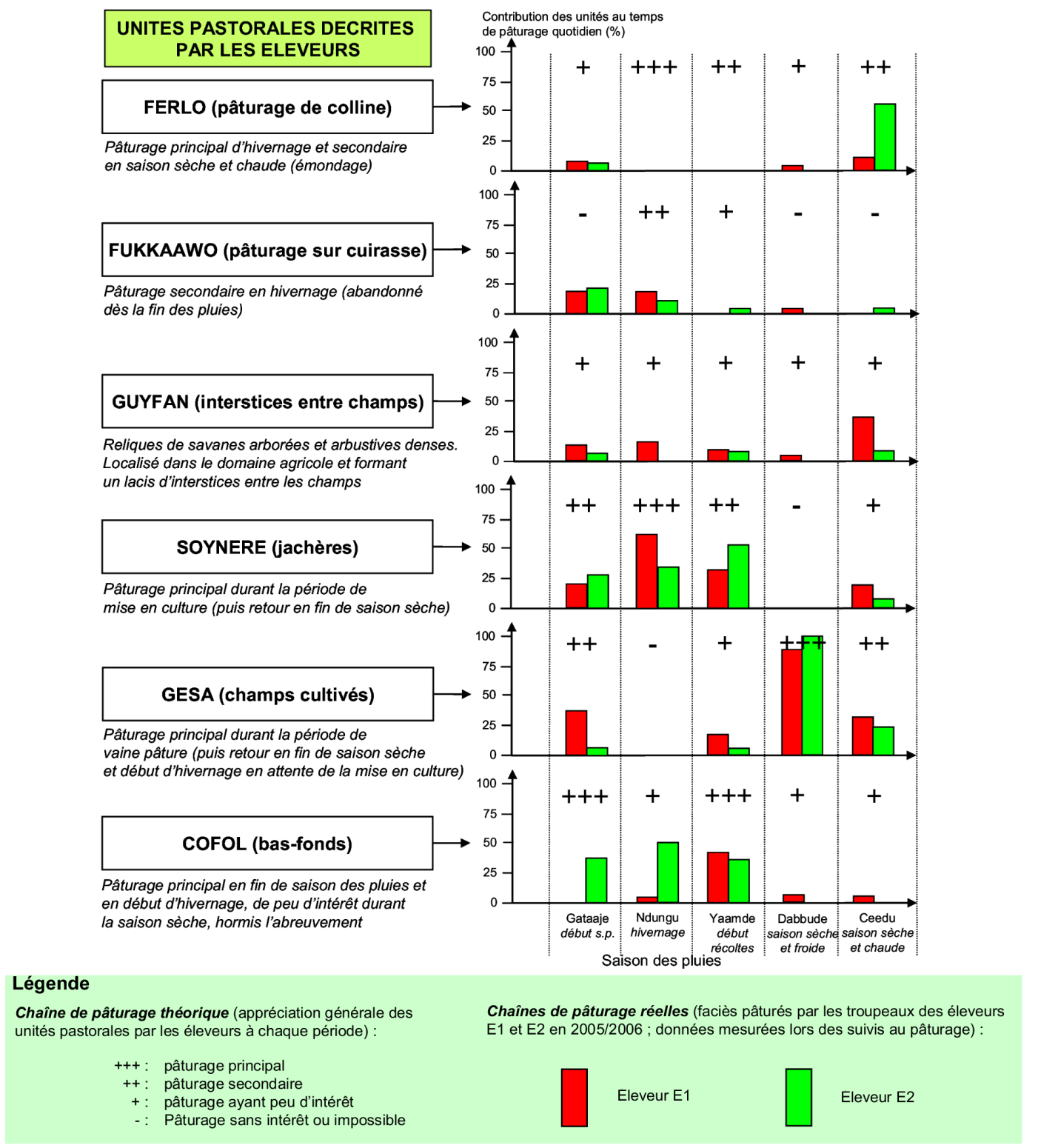

Fig. 2. Chaînes de pâturage réelles des éleveurs E1 et E2.

\section{De l'analyse à la préparation de l'intervention}

Les suivis des troupeaux au pâturage ont enfin permis de confirmer le caractère stratégique de certains faciès de pâturage et de dresser, selon les périodes, une liste de faciès principaux et secondaires sur lesquels les éleveurs comptent pour nourrir leur troupeau (Tab. 5). À l'échelle du territoire de Koumbia-Waly, l'espace agricole (gesa et soynere) couvre environ $53 \%$ du territoire villageois, les formations ripicoles le long des bas-fonds (cofol) environ $10 \%$, les pâturages de collines ferlo environ $10 \%$ et le fukkaawo $13 \%$ (d'après Blanchard, 2005), mais ces estimations devront être confirmées par des travaux cartographiques plus précis. Hormis la zone de culture, les unités pastorales identifiées sont très fragmentées.
Ces faciès doivent être préservés en priorité; en outre, pour que les troupeaux puissent les atteindre au moment opportun, la circulation du bétail doit être garantie entre les unités, car elles ne sont pas disposées de façon continue dans l'espace (cf. Fig. 3). La réglementation de la vaine pâture doit être reprécisée. De telles dispositions (préservation, circulation) pourront être intégrées dans la convention locale, avec à l'appui des règles de gestion instituées, des jeux de cartes calées sur le calendrier pastoral et les nomenclatures des faciès reconnues par les praticiens. Des aménagements spécifiques à chaque unité pastorale pourront être discutés et mis en place avec la population du village pour les valoriser et réduire les conflits. 
Tableau 4. Pratiques circonstancielles de pâturage des éleveurs E1 et E2.

\begin{tabular}{|c|c|c|}
\hline Période & Ajustements par opportunité & Ajustements par contrainte \\
\hline \multicolumn{3}{|c|}{ Éleveur 1} \\
\hline $\begin{array}{l}\text { Gataaje } \\
\text { Début d'hivernage }\end{array}$ & $\begin{array}{l}\text { Pâturage de fukkaawo (cuirasse) permis } \\
\text { par une pousse de l'herbe résultant de } \\
\text { pluies précoces }\end{array}$ & Cofol (bas-fond) trop éloigné \\
\hline $\begin{array}{l}\text { Ndungu } \\
\text { Hivernage }\end{array}$ & - & $\begin{array}{l}\text { Ferlo (colline) trop éloigné et inaccessible } \\
\text { en raison des cultures }\end{array}$ \\
\hline $\begin{array}{l}\text { Yaamde } \\
\text { Début de récoltes }\end{array}$ & $\begin{array}{l}\text { Récoltes précoces permettant un retour } \\
\text { sur gesa (résidus de cultures) }\end{array}$ & $\begin{array}{l}\text { Ferlo (colline) trop éloigné et inaccessible } \\
\text { en raison des cultures }\end{array}$ \\
\hline $\begin{array}{l}\text { Dabbude } \\
\text { Saison sèche et froide }\end{array}$ & - & - \\
\hline $\begin{array}{l}\text { Ceedu } \\
\text { Saison sèche et chaude }\end{array}$ & $\begin{array}{l}\text { Installé dans des champs, le troupeau } \\
\text { pâture les guyfan (interstices), soynere } \\
\text { (jachères), gesa (résidus de cultures) }\end{array}$ & $\begin{array}{l}\text { Le ferlo (colline), trop éloigné, est remplacé } \\
\text { par les guyfan (interstices) plus accessibles }\end{array}$ \\
\hline \multicolumn{3}{|c|}{ Éleveur 2} \\
\hline $\begin{array}{l}\text { Gataaje } \\
\text { Début d'hivernage }\end{array}$ & $\begin{array}{l}\text { Pâturage de fukkaawo (cuirasse) permis } \\
\text { par une pousse de l'herbe résultant de } \\
\text { pluies précoces }\end{array}$ & - \\
\hline $\begin{array}{l}\text { Ndungu } \\
\text { Hivernage }\end{array}$ & $\begin{array}{l}\text { Pâturage du cofol (bas-fond) } \\
\text { exceptionnellement peu inondé }\end{array}$ & $\begin{array}{l}\text { Ferlo (colline) délaissé car la pousse de } \\
\text { l'herbe est insuffisante }\end{array}$ \\
\hline $\begin{array}{l}\text { Yaamde } \\
\text { Début de récoltes }\end{array}$ & - & $\begin{array}{l}\text { Ferlo (colline) délaissé car la pousse de } \\
\text { l'herbe est insuffisante }\end{array}$ \\
\hline $\begin{array}{l}\text { Dabbude } \\
\text { Saison sèche et froide }\end{array}$ & - & - \\
\hline $\begin{array}{l}\text { Ceedu } \\
\text { Saison sèche et chaude }\end{array}$ & $\begin{array}{l}\text { Proche du ferlo (colline), il y exploite les } \\
\text { fourrages ligneux (pratique de } \\
\text { l'émondage) }\end{array}$ & - \\
\hline
\end{tabular}

Tableau 5. Pâturages principaux et secondaires confirmés par les suivis.

\begin{tabular}{l|l|l}
\hline Périodes du calendrier pastoral & Pâturages principaux & Pâturages secondaires \\
\hline $\begin{array}{l}\text { Gataaje } \\
\text { Début de saison des pluies (mai-juin) }\end{array}$ & cofol & soynere, gesa \\
\hline $\begin{array}{l}\text { Ndungu } \\
\text { Saison des pluies (juil.-sep.) }\end{array}$ & soynere, ferlo & fukkaawo, guyfan \\
\hline $\begin{array}{l}\text { Yaamde } \\
\text { Début de récoltes (octobre) }\end{array}$ & cofol & soynere, ferlo \\
\hline $\begin{array}{l}\text { Dabbude } \\
\text { Saison sèche et froide (nov.-janv.) }\end{array}$ & gesa & tous les autres faciès \\
\hline $\begin{array}{l}\text { Ceedu } \\
\text { Saison sèche et chaude (fév.-avril) }\end{array}$ & Pas de véritable pâturage principal & ferlo, gesa \\
\hline
\end{tabular}

\section{Un cadre d'analyse original pour l'étude des pratiques?}

\section{Un éclairage différent sur les pratiques}

En agronomie et en zootechnie systémique, l'analyse des pratiques débute classiquement par une étude de la diversité des situations, débouchant sur des typologies élaborées sur la base d'entités définies par les experts. Les types sont ensuite caractérisés par un jeu de variables définies elles aussi a priori, pour aboutir à l'élaboration des références sur les pratiques.
Avec le cadre d'analyse proposé, l'étude de la diversité est effectuée en référence aux systèmes de classification reconnus par les producteurs (entités du savoir local). Les entités ainsi définies sont caractérisées par des variables reconnues par les producteurs (variables de description, de fonctionnalité et de risque) et complétées au besoin par un jeu de variables choisies par les experts. Ainsi, les références sur les pratiques sont élaborées en fonction des entités et des variables utilisées par les producteurs. Elles ont du sens pour les acteurs de terrain et les scientifiques. 


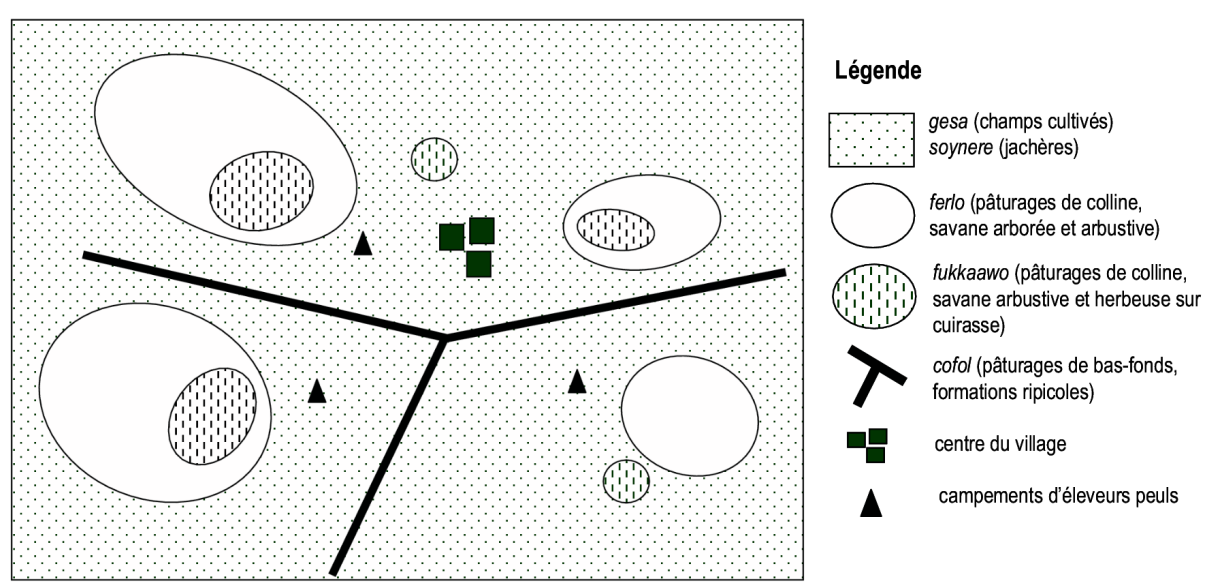

Fig. 3. Schéma de la répartition spatiale des principaux pâturages.

Le calendrier pastoral des éleveurs est plus complexe que celui classiquement présenté, basé sur trois ou quatre périodes (Petit, 2000 ; Ickowicz et Mbaye, 2001 ; Botoni, 2003 ; Augusseau, 2007). Les deux intersaisons (gataaje et yaamde) marquent des moments critiques en termes de transition fourragère, de décision à prendre (départ/retour en transhumance, négociation de contrat de pâture...) et de risques encourus. Il nous paraît crucial de les intégrer à l'analyse des pratiques pour comprendre leur logique, notamment pour analyser la flexibilité des systèmes (Dedieu et al., 2008).

Les unités pastorales reconnues par les éleveurs ont une base fonctionnelle cohérente avec les pratiques de conduite des troupeaux au pâturage et ne correspondent pas aux critères de classification des faciès de savanes définis par les écologues (Aubreville, 1957), souvent repris par les pastoralistes (Botoni, 2003). Dans les zones agropastorales, l'éleveur évalue la valeur d'un pâturage par un coût d'opportunité global intégrant la valeur alimentaire, l'accessibilité à l'eau et les risques de conflit et de maladie, alors que les experts ne retiennent souvent que la valeur pastorale. L'art de la conduite du troupeau au pâturage réside dans la capacité à satisfaire les besoins alimentaires des animaux en minimisant les risques de conflit liés à l'exploitation séquentielle et partagée des espaces.

\section{Une production de savoirs actionnables}

Les références produites ont du sens pour les acteurs de terrain, car elles se basent sur la façon dont ils se représentent les choses. Élaborées à partir de la nomenclature des praticiens, elles constituent de notre point de vue les éléments d'un langage commun nécessaire pour susciter le dialogue tout en évitant les malentendus. En favorisant l'échange de points de vue entre praticiens sur les problèmes à traiter, l'identification des solutions devrait en être facilitée. Cela leur confère un caractère actionnable au sens d'Avenier et Schmitt (2007).
Dans le cadre de cette étude, elles éclairent les dispositions à prendre en matière de préservation des unités pastorales et de mobilité du bétail. Pour chaque saison, les principales unités pastorales sont connues, ainsi que la polarité des déplacements des troupeaux et les raisons qui les sous-tendent. Pour préserver la flexibilité des systèmes et donc leur durabilité dans un environnement incertain, la convention locale contribuera à sécuriser les unités pastorales principales et secondaires et à réglementer les déplacements des troupeaux entre ces unités et vers les points d'eau. Des documents cartographiques figurant ces unités pastorales pourront être élaborés pour chaque période et auront ainsi plus de chance d'être compris et utilisés pour organiser la conduite des troupeaux au pâturage sur le territoire de Koumbia-Waly.

\section{Conclusion}

Ce travail propose un cadre d'analyse des pratiques prenant en compte les savoirs techniques locaux. Il est applicable à tout type d'études sur les pratiques et il est facile à mettre en œuvre par des agronomes et des zootechniciens pour comprendre les fondements et la diversité des pratiques.

Il permet de produire des références ayant du sens pour les acteurs de terrain et les scientifiques. Nous avons d'ores et déjà constaté, lors des restitutions, que les références présentées suscitaient le débat, la réflexion et la compréhension des points de vue réciproques, ce qui est de bon augure pour la concertation, la prise d'initiatives et leur mise en mouvement, autrement dit pour l'action et l'innovation. Aussi, le temps consacré à l'étude des savoirs locaux, en préalable à l'étude des pratiques, permettra de progresser plus vite dans la phase d'intervention, grâce au caractère actionnable des références établies.

Ce travail reste néanmoins perfectible, notamment au niveau de la caractérisation des indicateurs, dont le 
caractère tacite rend leur expression difficile tant ils se trouvent enracinés dans les routines des praticiens. L'explicitation des indicateurs mériterait d'être approfondie.

Des analyses comparées de situations pourraient être développées pour produire des connaissances à caractère générique sur les variables et les indicateurs de caractérisation des entités du savoir local réellement utilisés par les praticiens. La comparaison des situations pourrait ainsi être conduite tant dans l'espace, pour préciser les contenus des fonds communs de connaissances et leurs variantes, que dans le temps, pour étudier le caractère évolutif des savoirs locaux (transformations des systèmes de classification, évolution des variables et des indicateurs pris en compte...).

\section{Remerciements}

Les auteurs remercient le projet DURAS (GFAR, MAE) et l'ATP CIROP (Cirad) pour leur soutien financier et scientifique à cette initiative de recherche sur les savoirs locaux et la recherche en partenariat.

\section{Références}

Akrich, M., Callon, M., Latour, B., 2006. Sociologie de la traduction, Paris, École des mines.

Aubreville, A., 1957. Accord à Yangambi sur la nomenclature des types africains de végétation, Bois et forêts des tropiques, 51, 23-27.

Augusseau, X., 2007. Évolution des systèmes agropastoraux dans le Sud-Ouest du Burkina Faso : interactions et dynamiques territoriales. Thèse de doctorat en géographie et aménagement de l'espace, Université Montpellier III, Montpellier.

Avenier, M.-J., Schmitt, C., 2007. La Construction de savoirs pour l'action, Paris, L'Harmattan.

Blanchard, M., 2005. Relations agriculture-élevage en zone cotonnière : territoire de Koumbia et Waly, Burkina Faso. Mémoire de DESS, Université Paris XII, Créteil.
Botoni, H.E., 2003. Interactions élevage-environnement : dynamique des paysages et évolution des pratiques pastorales dans les fronts pionniers du Sud-Ouest du Burkina Faso. Thèse de doctorat en biologie des populations et écologie, Université Montpellier III, Montpellier.

Cyrulnik, B., 1995. La Naissance du sens, Paris, Hachette.

Darré, J.-P., Mathieu, A., Lasseur, J., 2004. Le Sens des pratiques : conceptions d'agriculteurs et modèles d'agronomes, Paris, INRA Éditions.

Dedieu, B., Chia, É., Leclerc, B., Moulin, C.-H., Tichit, M. (Eds), 2008. L'Élevage en mouvement, Paris, Quæ.

Diallo, M.A., 2006. Savoirs locaux et pratiques de conduite des troupeaux au pâturage : élaboration d'une méthode d'étude. Mémoire de DEA, LERNSE/IDR/UPB, Bobo-Dioulasso (Burkina Faso).

Diallo, M.A., 2007. Savoirs techniques locaux et conduite des troupeaux au pâturage : le cas des éleveurs de Koumbia et de Kourouma, Bobo-Dioulasso, CIRDES.

Dupré, G., 1991. Savoirs paysans et développement, Paris, Karthala/ORSTOM.

Kagoné, H., 2000. Gestion durable des écosystèmes pâturés en zone nord-soudanienne du Burkina Faso. Thèse de doctorat, Faculté universitaire des sciences agronomiques de Gembloux, Gembloux.

Kiéma, S., 2007. Élevage extensif et conservation de la diversité biologique dans les aires protégées de l'Ouest burkinabé : arrêt sur leur histoire, épreuves de la gestion actuelle, état et dynamique de la végétation. Thèse de doctorat en physiologie et biologie des organismes, populations et interactions, Université d'Orléans, Orléans.

Ickowicz A., Mbaye M., 2001. Forêts soudaniennes et alimentation des bovins au Sénégal : potentiel et limites, Bois et forêts des tropiques, 55, 270, 47-61.

Liu, M., 1997. Fondements et pratiques de la recherche-action, Paris, L'Harmattan.

Olivier de Sardan, J.-P., 1996. Anthropologie et développement, Paris, Karthala.

Petit, S., 2000. Environnement, conduite des troupeaux et usage de l'arbre chez les agropasteurs peuls de l'Ouest burkinabé. Approche comparative et systématique de trois situations: Barani, Kourouma, Ouangolodougou. Thèse de doctorat en géographie, Université d'Orléans, Orléans.

Scoones, I., Thompson, J., 1999. La Reconnaissance du savoir rural, Paris, CTA/Karthala.

Vall, É., Dugué, P., Blanchard, M., 2006. Le tissage des relations agriculture-élevage au fil du coton, 1990-2005, Cahiers Agriculture, 15, 1, 72-79.

Reçu le 15 mai 2007. Accepté le 5 février 2009. 